\title{
Helical Coherent Structures in the Wake of Solitary Internal Waves Breaking on the Continental Slope
}

\author{
Alexander Soloviev ( $\nabla$ soloviev@nova.edu ) \\ Nove Southeastern University https://orcid.org/0000-0001-6519-1547 \\ Breanna Vanderplow \\ Nova Southeastern University \\ Cayla Dean \\ Nova Southeastern University
}

Physical Sciences - Article

Keywords: Stratified Coastal Waters, 3D Cascade of Energy, Computational Fluid Dynamics Model, Counterrotating Helices, Sediment Transport

Posted Date: November 2nd, 2020

DOI: https://doi.org/10.21203/rs.3.rs-83862/v1

License: (c) (1) This work is licensed under a Creative Commons Attribution 4.0 International License. Read Full License 


\title{
Helical Coherent Structures in the Wake of Solitary Internal Waves Breaking on the Continental Slope
}

\author{
Alexander V. Soloviev, Breanna Vanderplow, Cayla W. Dean \\ ${ }^{1}$ Halmos College of Natural Sciences and Oceanography, Nova Southeastern University, Dania \\ Beach, Florida 33004, USA
}

\section{Abstract}

Mixing caused by the solitary internal waves or solitons in stratified coastal waters is a primary cause of sediment resuspension and transport. Theoretical, experimental, and modeling studies of solitons have focused on nonlinear wave dynamics to explain their main features. However, the 3D cascade of energy from breaking internal wave solitons to turbulence and mixing in the wave induced wake has received less attention. Previous numerical simulations have revealed that internal wave breaking involves the lobe-cleft instability typical of gravity currents, resulting in longitudinal rolls ${ }^{1-3}$. Recently, a group at the Scripps Institution of Oceanography measured the fine structure of internal wave solitons in the coastal waters off California using the utility of fiber optic distributed temperature sensing (DTS) to gather such observations and observed the longitudinal rolls ${ }^{4,5}$. Here, we present the evidence that these structures are counter rotating helices. The concept of helicity ${ }^{6,7}$ as a topological invariant and a measure of the lack of mirror symmetry of the flow can explain the helical nature of these coherent structures. Both observational and modeling results are consistent with this theoretical conjecture. We assume the discovered helical structures are a significant component in the mixing, formation of nepheloid layers, and property exchanges caused by solitary internal waves breaking on continental slopes. 


\section{Introduction}

Internal wave solitons are a fascinating feature of ocean dynamics. An internal wave soliton is a train of nonlinear solitary internal waves with the highest amplitude wave in the lead. The wavelength of individual waves in the waveform ranges from tens of meters to tens of kilometers with amplitudes ranging from a few to tens of meters ${ }^{8-12}$. In stratified coastal waters, turbulence caused by internal wave solitons is a primary source of mixing ${ }^{13-15}$, affecting marine ecosystems, sediment resuspension and transport, and formation of nepheloid layers (Supplementary Video). Turbulence is a complex multi-scale phenomenon associated with chaotic motion and some degree of coherence and self-organization depending on the environmental conditions. The study of internal wave breakdown in shallow water has proceeded through a combination of theory,

numerical modeling, laboratory experiments, and field observations ${ }^{9-15}$. Turbulence and coherent structures developing in the wake of breaking internal waves have a substantial effect on the sediment transport in the bottom boundary layer, formation of nepheloid layers, and nutrient fluxes. Direct numerical simulations ${ }^{1-3}$ have revealed that internal wave breaking involves the lobe-cleft instability typical of gravity currents, resulting in longitudinal rolls. Recently, a group at the Scripps Institution of Oceanography measured the fine structure of internal wave solitons in the coastal waters off California using the utility of fiber optic distributed temperature sensing (DTS) to gather such observations and observed the longitudinal streaks ${ }^{4,5}$. Physics of these longitudinal streaks, however, has never received explanation. Here, we present the evidence that these streaks are coherent counter rotating helices. 
Oceanographic measurements on the California shelf have shown that after transitioning into nonlinear waves of elevation, the solitary internal waves begin to form a spatially coherent pattern extended behind the shoaling waves, which resembled the results of numerical modeling ${ }^{1-3}$. The cascade of energy from the internal wave field to turbulence evidently included spatially coherent organized structures. Our work seeks to provide a theoretical explanation of these coherent structures. To understand the origin of the structures, we have developed a 3D high-resolution model of an internal wave soliton breaking on the continental slope resembling the California Shelf using computational fluid dynamics ANSYS Fluent software. Subsequently, we interpreted the field and modeling results in the context of the Moffatt's theory of helicity ${ }^{7,8}$. We modeled the internal wave soliton breaking on the continental slope of California with a an ANSYS Fluent computational fluid dynamics model. The model settings are described in "Materials and Methods". The continental slope angle and depth range are like those in the experimental area on the California Shelf ${ }^{4,5}$. The numerical model generated three internal solitary waves. To mimic the experimental results obtained on the California Shelf, we plotted the difference of temperature from two successive model time steps with a $5 \mathrm{~s}$ time difference. The result of this subtraction is shown in Figure $1 \mathrm{~b}$ - the model pattern closely resembles observations $^{4,5}$. In particular, the top view reveals coherent streaks developing behind solitary wave fronts, which are similar those observed in the experiment on the California Shelf. The computational model shows that these coherent streaks appear to be counterrotating helices with the axis oriented in the direction of the internal wave propagation (Fig. 2a, b, and Fig. 3). 

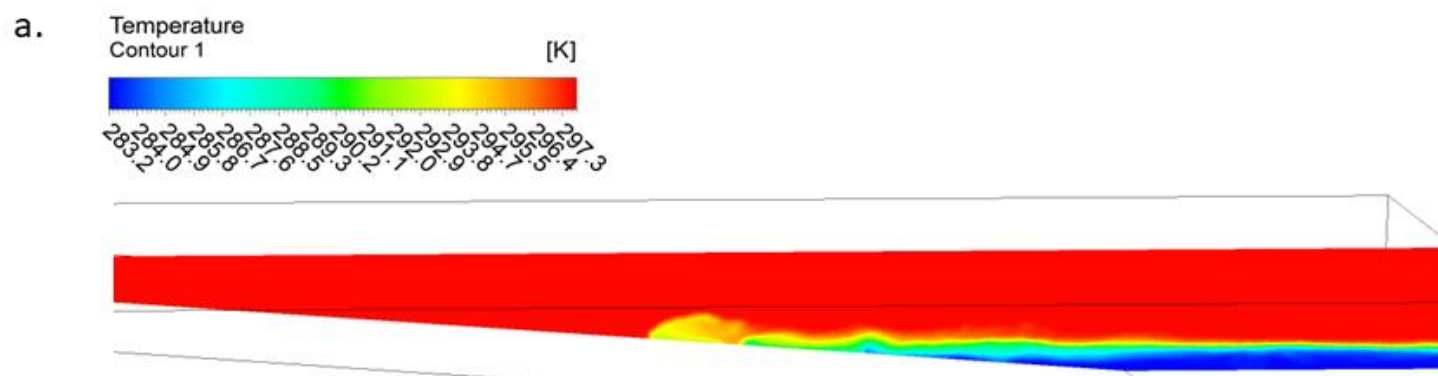

\section{$\stackrel{1}{\circ}$}

b.

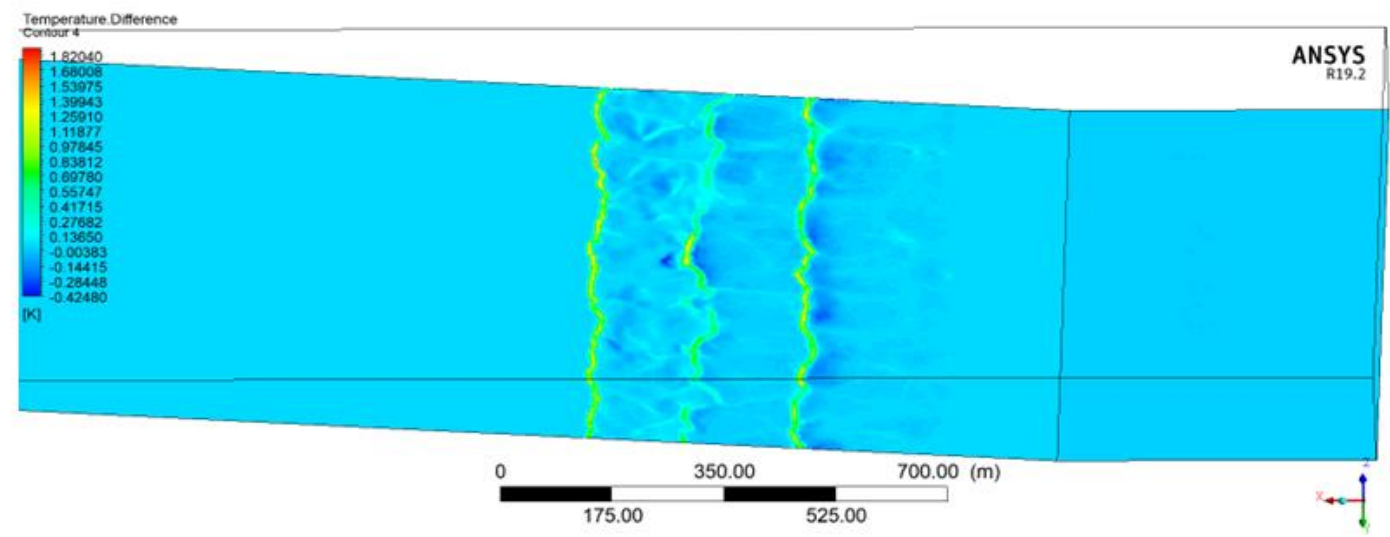

Figure 1. Side (a) and top (b) contour views of an internal soliton breaking on the continental slope like that in the California experiment ${ }^{4,5}$.

Both field data and the computational experiment show that the fronts of breaking solitary internal waves are somewhat meandrous and spatially inhomogeneous, in part due to coherent helical motions induced by the front. This factor may affect the interpretation of singleinstrument observations of propagating solitary waves.

Field observations enhanced with adequate numerical modeling provided an important insight into the dynamics of solitary internal waves. To our best knowledge, the helical coherent structures in the turbulent wake associated with breaking solitary waves have not been previously reported. These helical structures result in intense near-bottom mixing (Fig. 2). 


\section{Theoretical Considerations}

In this section, we offer a theoretical explanation of the helical phenomenon. Our hypothesis is that the source of the generation of helices is the shear induced by solitary internal waves in the near-bottom layer of the ocean. Helical motions can be represented by helicity ${ }^{6}$, which is defined by $H=\int \mathbf{u} \cdot \boldsymbol{\omega} d V$, where $\mathbf{u}(\mathbf{x}, t)$ is the velocity field, $\boldsymbol{\omega}(\mathbf{x}, t)=\nabla \times \mathbf{u}$ is the localized vorticity distribution in a fluid of infinite extent; and, the integral is over all space. This integral is, like energy, an invariant of the Euler equations of ideal flow ${ }^{6}$. Helicity is a pseudoscalar, which has been linked to flow topology as a measure of the lack of mirror symmetry of the flow ${ }^{6,7}$.

In his fundamental work ${ }^{7}$, Moffatt considers a turbulent flow, governed by the unsteady NavierStokes equation, in the following form,

$$
\partial_{t} \mathbf{u}=-\nabla h+\mathbf{u} \times \boldsymbol{\omega}+v \nabla^{2} \mathbf{u}, \quad \nabla \cdot \mathbf{u}=0,
$$

and supposes that in some areas within the turbulent field the helicity density $|\mathbf{u} \cdot \boldsymbol{\omega}|$ is maximal or near maximal, so that $\mathbf{u}$ is nearly parallel to $\pm \boldsymbol{\omega}$ (the vortex lines are frozen in the flow). In these areas the nonlinear term $\mathbf{u} \times \mathbf{\omega}$ in Eq. (1) is relatively small, and the nonlinear cascade of energy to smaller scales is also expected to decrease as a result. Correspondingly, the flow structure in regions where $|\mathbf{u} \cdot \boldsymbol{\omega}|$ is near maximal tends to persist coherently in the form of helices. Turbulent flow near a wall is intrinsically chiral (i.e., lacks reflection symmetry) and thus is favorable for the development of helical coherent structures. In concert with the law of conservation of angular momentum, these helical structures are counterrotating, which is indeed observed in computational results (Fig. 2a, b, and Fig. 3). 


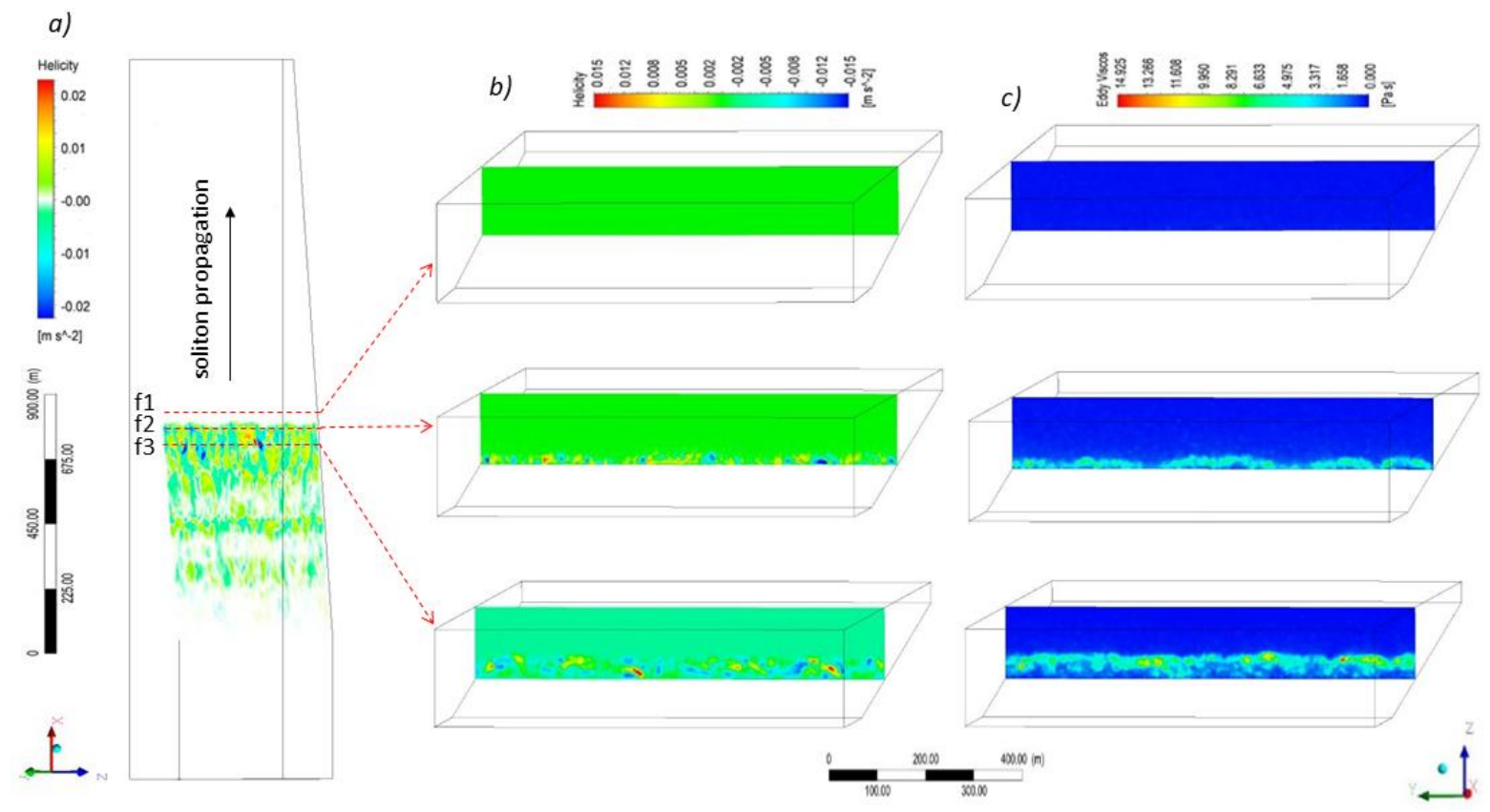

Figure 2. Coherent structures behind the wave front of a soliton breaking on the continental slope: (a) top view of helicity on a plane parallel to and elevated above the bottom by $10 \mathrm{~m}$ (f1, f2, and $\mathrm{f} 3$ are three planes, which are positioned before, within, and after the leading solitary wave, respectively), (b) contour plots of helicity, and (c) eddy viscosity.

This theoretical conjecture supports the idea that the shear induced by solitary internal waves in the near-bottom layer of the ocean is the source of the generation of helical coherent structures. The formation of helical coherent structures in the wall layer has been observed in laboratory experiments ${ }^{16}$ and reproduced in numerical models of oceanic overflows ${ }^{17}$ and spreading freshwater lenses interacting with wind stress ${ }^{18}$. 

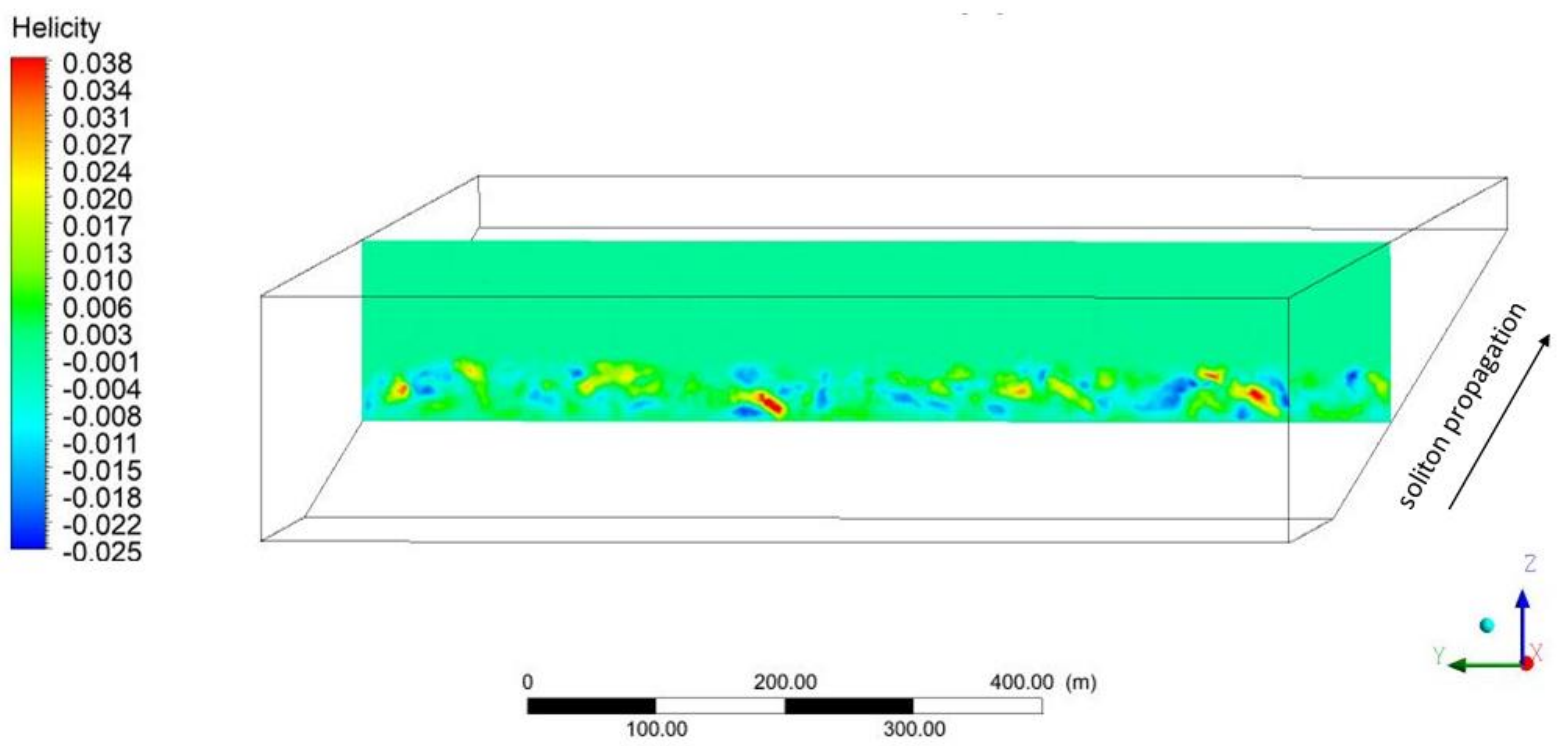

Figure 3. Contour plot of helicity from Figure 3b, plane f3, but in more detail. Helicity units are the same as in Figure $3 b\left(\mathrm{~m} \mathrm{~s}^{-2}\right)$. The intermittent sign of helicity features near the bottom is an indication of counterrotating spiraling structures with axes parallel to the direction of the soliton propagation.

\section{Conclusions}

Oceanographic measurements on the California shelf with the DTS system revealed coherent structures developing behind the leading front of the solitary internal wave breaking on the continental slope ${ }^{5}$. We have demonstration with a computer model that these coherent structures are counterrotating helices. The theoretical conjecture based on the concept of helicity ${ }^{3}$ suggests that the shear induced by solitary internal waves in the near-bottom layer of the ocean is the source of the generation of the pattern of counterrotating helices. Turbulent flow near a wall is intrinsically chiral (i.e., lacks reflection symmetry), which explains the development of helical coherent structures. The law of conservation of the angular momentum necessitates that the helices are counterrotating, which is also supported by the modeling results. Both observational 
and modeling results are consistent with this theoretical conjecture. We assume the discovered helical coherent structures are a significant component in the mixing, formation of nepheloid layers, and property exchanges caused by solitary internal waves breaking on continental slopes.

\section{Acknowledgements}

The authors are grateful to Andrew Lucas and Robert Pinkel (SIO) for fruitful discussion of the manuscript. This research was made possible by a grant from the Gulf of Mexico Research Initiative to Consortium for Advanced Research on Transport of Hydrocarbon in the Environment (CARTHE) and the Office of Naval Research Award N00014-18-1-2835.

\section{Literature}

1. Arthur, R.S. and O.B. Fringer, 2016. Transport by breaking internal gravity waves on slopes. Journal of Fluid Mechanics 789, 93-126.

2. Arthur, R. S. and O.B. Fringer, 2014 The dynamics of breaking internal solitary waves on slopes. Journal of Fluid Mechanics 761, 360-398.

3. Arthur, R. S., J.R. Koseff, \& O.B. Fringer, 2017. Local versus volume-integrated turbulence and mixing in breaking internal waves on slopes. Journal of Fluid Mechanics, 815, 169-198.

4. Lucas, A., and R. Pinkel, 2019. Fiber Optic Distributed Temperature Sensing Measurements of Breaking Internal Waves. 2019 IEEE/OES Twelfth Currents, Waves, turbulence Measurements and Applications Workshop (CWTMA). Catamaran Resort, San Diego, 10-13 March 2019.

5. Lucas, A., and R. Pinkel, 2020. Observations of a coherent instability in shoaling oceanic internal waves. Geophysical Research Letters. Submitted. 
6. Moffatt, H.K., 1969. The degree of knottedness of tangled vortex lines. Journal of Fluid Mechanics 35, 117-129.

7. Moffatt, H.K., 2014. Helicity and singular structures in fluid dynamics. PNAS 111, 36633670.

8. Jackson, C.R., J.C.B. da Silva, and G. Jeans, 2012. The generation of nonlinear internal waves. Oceanography 25(2), 108-123.

9. Stanton, T.P. and L. A. Ostrovsky, 1998. Observations of highly nonlinear internal solitons over the continental shelf. Geophysical Research Letters 25, 2695-2598.

10. Perry, R.B. and G.R. Schimke, 1965. Large amplitude internal waves observed off the north-west coast of Sumatra. Journal of Geophysical Research 70, 2319-24.

11. Farmer D.M., 1978. Observations of long nonlinear internal waves in a lake. Journal of Physical Oceanography 8, 63-73.

12. Boegman, L. and M. Stastna, 2019. Sediment Resuspension and Transport by Internal Solitary Waves. Annual Review of Fluid Mechanics 51,129-154.

13. Alberty, M. S., S. Billheimer, M. M. Hamann, C. Y. Ou, V. Tamsitt, A. J. Lucas, and M. H. Alford, 2017. A reflecting, steepening, and breaking internal tide in a submarine canyon. Journal of Geophysical Research - Oceans 122, 6872-6882.

14. Helfrich, K.R. and W. K. Melville, 2006. Long Nonlinear Internal Waves. Annual Review of Fluid Mechanics 38(1), 395-1,425.

15. Leichter, J.J., G. B. Deane, M. D. Stokes, 2005. Spatial and Temporal Variability of Internal Wave Forcing on a Coral Reef. Journal of Physical Oceanography 35, 1945 1962. 
16. M. Lesieur, M., 2008. Turbulence in fluids. Number 84 in Fluid Mechanics and its Applications. Springer, Dordrecht, 4. rev. and enlarged ed edition, OCLC: 255895179.

17. Özgökmen, T.M., P.F. Fischer, J. Duan, and T. Iliescu, 2004. Three-dimensional turbulent bottom density currents from a high-order nonhydrostatic spectral element model. Journal of Physical Oceanography 34(2), 2006-2,026.

18. Soloviev, A.V., S. Matt, and A. Fujimura, 2015: Three-Dimensional Dynamics of Freshwater Lenses in the Ocean's Near-Surface Layer. Oceanography 28(1), 142-149.

\section{Materials and Methods}

ANSYS FLUENT 19.2 was used to model the internal wave breaking on the continental slope. For further consideration of the theory behind FLUENT models, please see the ANSYS Fluent Theory Guide 19.2. (2018). The model was run in parallel on 54 processors on a Dell workstation. The model domain, created in ANSYS Workbench, involved a $2500 \mathrm{~m}$ long, $180 \mathrm{~m}$ high, and $800 \mathrm{~m}$ wide domain with a $4.4^{\circ}$ continental slope. The mesh resolution was set to $0.5 \mathrm{~m}$ in all directions, with a total of $18,884,491$ cells. Boundary conditions were set to zero shear on the lateral sides and top of the domain, and no slip on the bottom and slope of the domain.

We used a pressure-based, transient, $2^{\text {nd }}$ order implicit solver and a Large Eddy Simulation (LES) turbulence model with Wall Adapting Local Eddy (WALE) as the sub-grid scale model. The LES model resolves momentum, mass, and energy in large eddies, which are significantly affected by the geometry and boundary conditions of the model. The governing equations of the LES model are derived from time-dependent Navier-Stokes equations that are filtered in either Fourier (wave-number) space or configuration (physical) space. Operating conditions were set to $293.15 \mathrm{~K}, 9.81 \mathrm{~m} / \mathrm{s}$ gravity, and $101325 \mathrm{~Pa}$ atmospheric pressure. We 
specified the material in our domain as water with thermal conductivity $=0.6 \mathrm{~W} / \mathrm{m} \mathrm{K}^{-1}$, viscosity $\mu=0.001003 \mathrm{~kg} \cdot \mathrm{m}^{-1} \cdot \mathrm{s}^{-1}$, and specific heat $c p=4182 \mathrm{~J} \mathrm{~kg}^{-1} \mathrm{~K}^{-1}$. The density and temperature of water was set according to a user defined function (UDF) written in $\mathrm{C}^{++}$, which was compiled and initialized within Fluent. The UDF was used to set the initial density anomaly, which created the internal wave using a defined temperature and salinity at the bottom left corner of the domain. The model was initialized with a temperature anomaly of $14.6^{\circ} \mathrm{C}$ and a salinity anomaly of $1.25 \mathrm{psu}$, which resulted in a density anomaly of $4.5 \mathrm{~kg} / \mathrm{m}^{3}$. This anomaly then propagated into an internal wave soliton which broke on the continental slope. 


\section{Figures}

a. Temperature

Contour 1 [K]

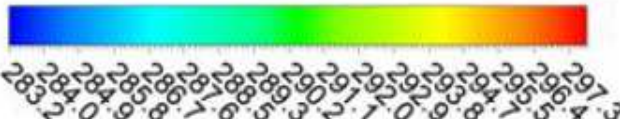

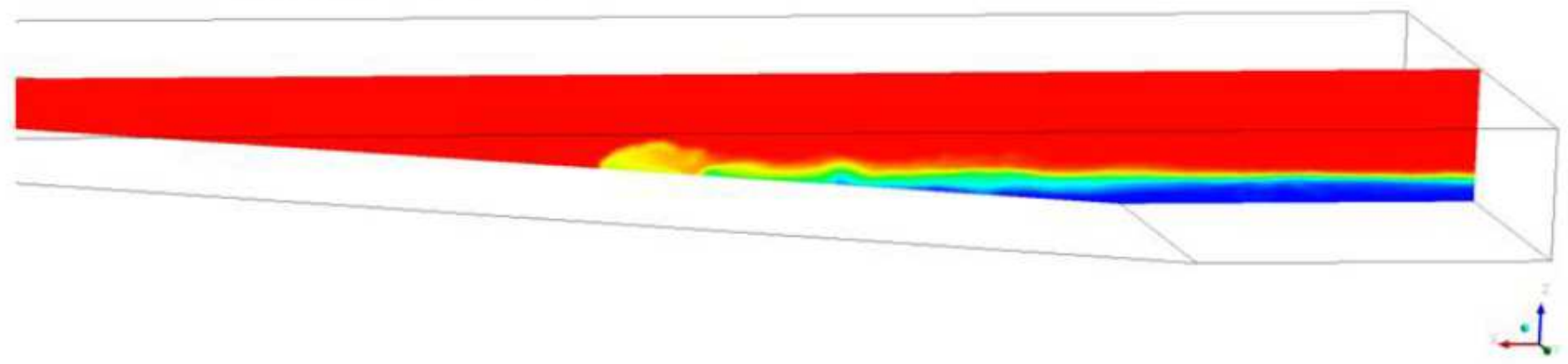

b. Temperature Deference

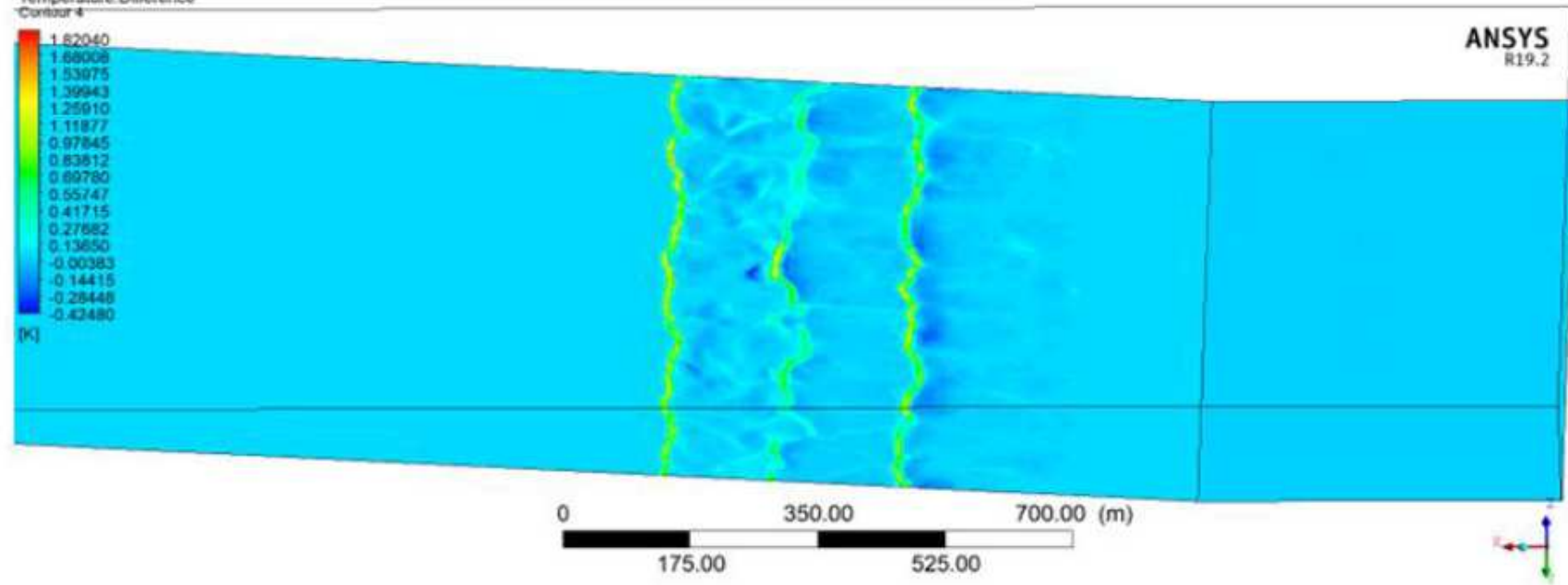

Figure 1

Side (a) and top (b) contour views of an internal soliton breaking on the continental slope like that in the California experiment4, 5 . 


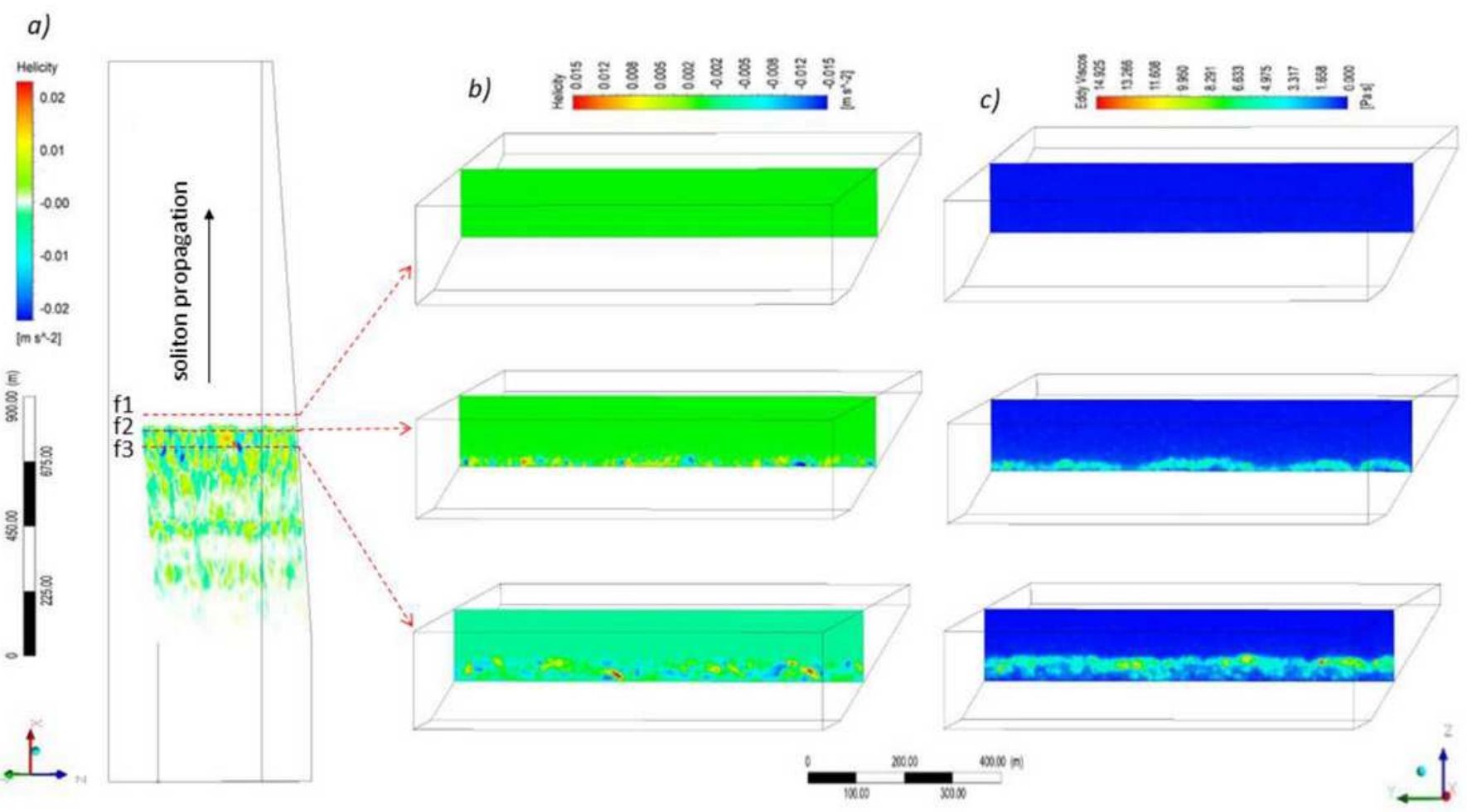

Figure 2

Coherent structures behind the wave front of a soliton breaking on the continental slope: (a) top view of helicity on a plane parallel to and elevated above the bottom by $10 \mathrm{~m}(\mathrm{f} 1, \mathrm{f} 2$, and $\mathrm{f} 3$ are three planes, which are positioned before, within, and after the leading solitary wave, respectively), (b) contour plots of helicity, and (c) eddy viscosity.
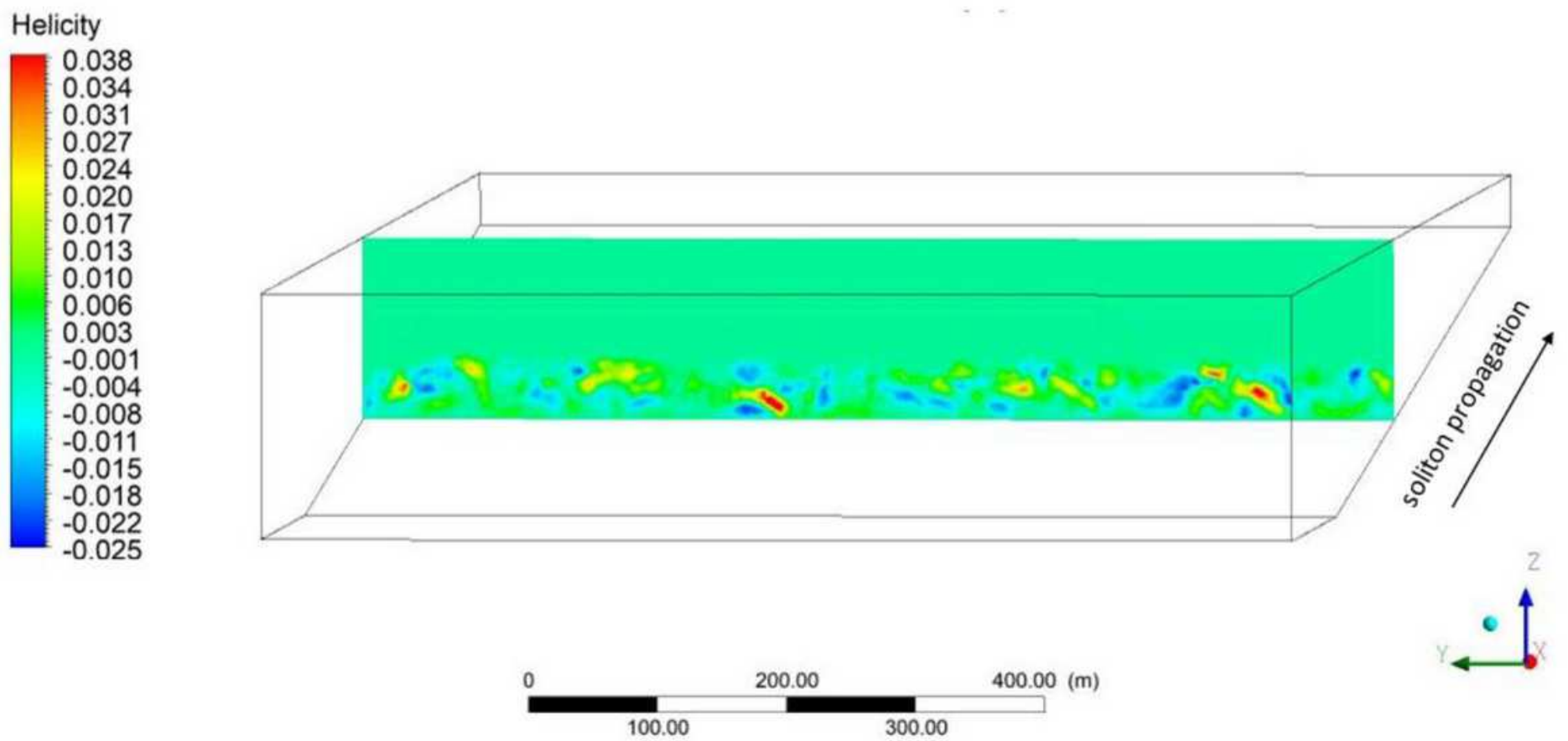


\section{Figure 3}

Contour plot of helicity from Figure $3 b$, plane $\mathrm{f} 3$, but in more detail. Helicity units are the same as in Figure $3 \mathrm{~b}(\mathrm{~m} \mathrm{~s}-2)$. The intermittent sign of helicity features near the bottom is an indication of counterrotating spiraling structures with axes parallel to the direction of the soliton propagation.

\section{Supplementary Files}

This is a list of supplementary files associated with this preprint. Click to download.

- internalwaverev1.mp4 